\title{
Sensibility Analysis of the Wen Zhutang Landslide Stability Based on Grey Correlation Analysis
}

\author{
Xin Chai ${ }^{a}$, Lei Nie ${ }^{b}$, Wenfei Mao $^{c}$, Min Zhang ${ }^{d}$ \\ College of Construction Engineering, Jilin University, Changchun 130026, China

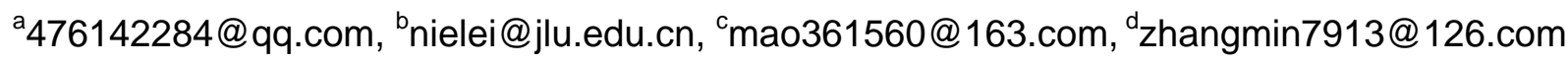

Keywords: landslide stability; grey correlation model; sensitivity.

\begin{abstract}
Grey correlation model is employed to analyze sensitivities of influencing factors for landslide stability in this paper. This model regards sensitive factors of the landslide stability as compare sequences, the corresponding landslide stability coefficients as reference sequences, makes the basic data dimensionless using top-difference transforming method and finally the grey correlative factors and grey correlative degrees which are indicators of sensitivity evaluation are computed. The model takes the sensibility analysis of a single planar landslide as an example, and in the sensibility analysis five influencing factors are considered, which are unit weight, cohesion, internal friction angle, seismic acceleration. The result shows that cohesion and internal friction angle of the sliding zone are the most susceptive factors influencing the landslide stability. Based on the result, the method of anchoring technology can be used to reinforce the landslide.
\end{abstract}

\section{Introduction}

Landslide is a common geological disasters in mountainous area, while China is a mountainous country with many landslide disasters, which cause great harm to construction and people's lives and property ${ }^{[1-3]}$. With the development of national economic development and construction, the frequency of landslide hazards in particular large-scale landslide disasters is increasing ${ }^{[4]}$.

Stability factor is an important parameter for the degree of landslide stability analysis. Is generally believed that the stability factor is greater than 1 , the landslide is in a safe state, and on the contrary, the landslide is in critical condition or state of insecurity. Many factors affect the stability of the landslide, mainly factors are topography, formation lithology, geological structure, seisms, shear strength index, groundwater level, human activities, etc. However, the influence degree of each factor on the landslide stability is different. At the time of the landslide stability analysis and evaluation, landslide stability coefficient can be easily obtained by a variety of methods under certain parameters, and can come with a stability coefficient changes with a parameter change rule. But in the actual project, due to the limitation of data volume and the order of magnitude, simply cannot evaluate the size of these factors ${ }^{[5-6]}$, so to find a suitable method for sensitivity analysis of these factors is particularly necessary.

Landslide stability factor sensitivity analysis methods can be divided into two categories. The first category is the multiple factors variation analysis, such as orthogonal design, uniform design, and BP neural network method. These methods require a certain amount of accurate data as training samples, and for the combination of engineering practice, some of these factors is not exist. The second category is the single factor variation analysis, a common way to proceed is first-selected indicators, and then determine the indexes influencing factors and the scope of their changes. Calculating, assuming one of the factors change and other factors remain the same, the relation curve plotted in the two-dimensional coordinates for indicators and changes of this factor, and so on. Until all the uncertainties in the calculation finish, and compare the index range along with the change of various factors, which have large range ability is called sensitive factor. This method is simple, however, due to various dimensionless parameters are inconsistent, and is not directly comparable. The order of magnitude of each parameter value vary widely, it is concluded that the variation of stability coefficient is large, meanwhile the safety factor and influence factors is not characterized by a linear relationship, its limitations are very obvious. In this paper, the grey correlation analysis method in 
grey system theory is used for a typical single planar landslide sensitivity factors analysis of its stability, which can overcome the shortage of single factor analysis method and find out the dominant factors influencing landslide stability, thus putting forward to targeted treatment measures. In this way can make it great significance for scheme optimization of landslide disaster prevention and treatment.

\section{Landslide environmental profiles}

\subsection{Engineering geological conditions}

Wen Zhutang landslide located in the northwest Wangcheng District of Changsha City, Hunan Province, and landslide area is given priority to low hilly landscape with tectonic denudation, and the Guchong Reservoir is in its front edge. The landslide is located upstream of the reservoir on the right bank slope, the peak elevation is $193 \mathrm{~m}$, undulating terrain. The slope roughly east-west strikes, leans to the riverbed, tendency to $\mathrm{NW} 356^{\circ}$, relatively flat, the average angle is about $28^{\circ}$. According to survey data which show that formation is predominantly thick-bedded weathered-strongly weathered politic siltstone, sandstone. Its layer thickness $30-50 \mathrm{~cm}$, thickness greater than $50 \mathrm{~m}$, local with mudstone, occurrence of $\mathrm{NW}_{353^{\circ}} \angle 22^{\circ}, \gamma=20 \mathrm{kN} / \mathrm{m}^{3}, \mathrm{c}=50 \mathrm{kPa}, \varphi=35^{\circ}$. Groundwater depth $10 \mathrm{~m}$, substantially parallel to the terrain lines. On the leading edge of landslide is bulge phenomenon, trailing edge to produce pulling cracks, along the mudstone interlayer sliding with a thickness of about $30 \mathrm{~cm}$. According to the shear test and inverse calculation of limit state for the final shear strength of the sliding surface which determines $c=18 \mathrm{kPa}, \varphi=23^{\circ}$. The regional seismic intensity of VI degrees, the earthquake peak acceleration value of $0.05 \mathrm{~g}$. Reservoir annual average water level is $98 \mathrm{~m}$, the maximum design water level is $113 \mathrm{~m}$. The engineering geological profile of landslide section is shown in Figure 1.

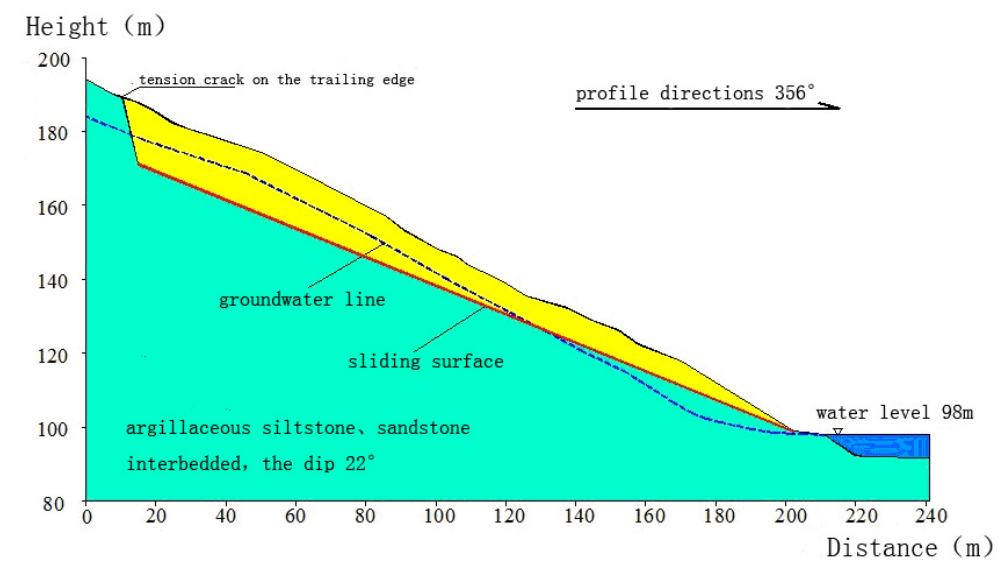

Fig.1 The engineering geological profile of landslide section

Wen Zhutang and slide is located in the east coast of White Horse River valley, concave bank of the river direction, forming a typical "Sicyon homologous" landslide topography with the northeast hilly slope crest. There is a small quarry near the western part in which engineering activities also contributes to the deformation and displacement of the landslide. Through field investigation found that the lower part rock of the landslide crushed more, and the reservoir bank slope concrete spray surface protection has obvious cracks, quarry access roads also appear shear cracks varying degrees. Geological disasters caused by landslide in the reservoir area will directly affect the developed eco-tourism projects. Since the landslide is located in the Guchong Reservoir range, once the landslide overall slides into reservoir, waves generated will cause serious harm to the reservoir dam, threatening the safety of downstream reservoirs simultaneously.

\section{The basic principles and methods of Gray Correlation Analysis}

\subsection{Grey Correlation Analysis Principle}

The Grey System Theory by Deng Julong is first proposed in the early 1980s, after nearly 30 years of development, it has penetrated into many areas and also has a widespread application in 
geotechnical engineering ${ }^{[7-10]}$. Grey correlation analysis is an integral part of Grey System Theory, It can make use of some limited data, more accurately search for various changeable factors (comparative actors) and the correlation between the reference factors (expressed as correlation degrees). The greater relevance is, indicating relatively stronger correlation between the comparative actors and reference factors. The basic principle of the gray correlation analysis is: Examine the microscopic or macroscopic geometric proximity relationship between various factors, analyze and determine the influence degree between various factors or the influence of several sub-factors for reference factors. Thus it can be very intuitive to measure correlation degree between factors based on sorting the quantitative correlation.

\subsection{Grey Correlation Analysis Methods}

The specific steps of grey correlation analysis ${ }^{[11-12]}$ can be divided into five steps, as shown in Fig.2.

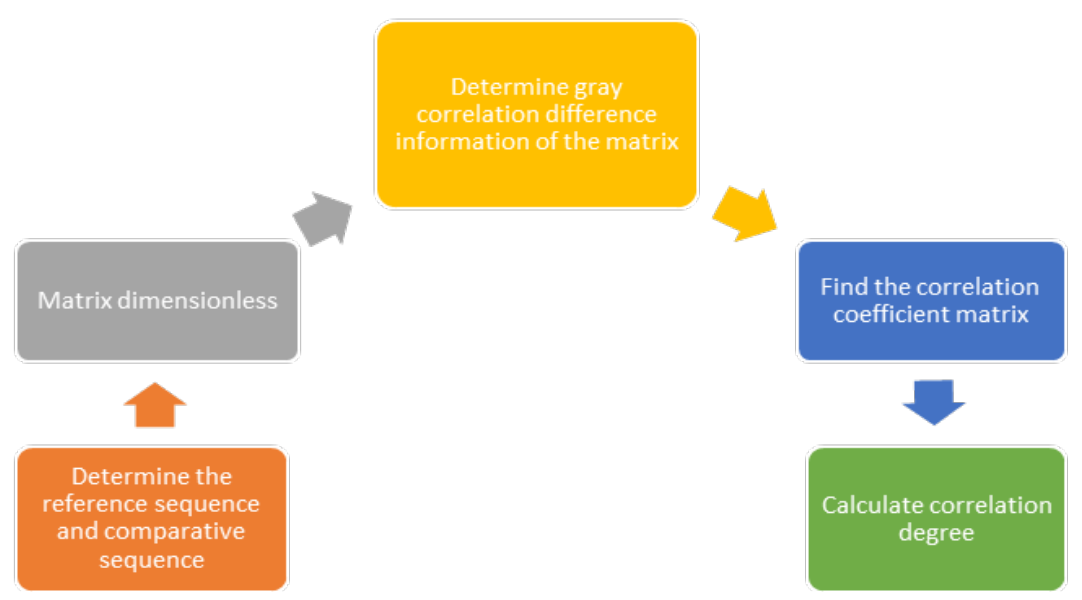

Fig.2 Flow chart of grey correlation degree analysis

Step one: Determine the reference sequence and comparative sequence. Select landslide susceptibility factors such as shear strength, underground water level, earthquakes and other factors as comparative sequence $X, X=\left[X_{1}, X_{2}, \ldots, X_{m}\right]^{\mathrm{T}}$, the corresponding stability coefficient of landslide is used as a reference sequence $Y, Y=\left[Y_{1}, Y_{2}, \ldots, Y_{m}\right]^{\mathrm{T}}$. Among them, the sequence $X, Y$ of each factor has a number of values, $X_{i}=\left[x_{i}(1), x_{i}(2), \ldots, x_{i}(\mathrm{n})\right], Y_{i}=\left[y_{i}(1), y_{i}(2), \ldots, y_{i}(\mathrm{n})\right], X, Y$ written in matrix form as follows:

$$
\begin{gathered}
X=\left[\begin{array}{c}
X_{1} \\
X_{2} \\
\vdots \\
X_{\mathrm{m}}
\end{array}\right]=\left[\begin{array}{cccc}
x_{1}(1) & x_{1}(2) & \cdots & x_{1}(\mathrm{n}) \\
x_{2}(1) & x_{2}(2) & \cdots & x_{2}(\mathrm{n}) \\
\vdots & \vdots & \ddots & \vdots \\
x_{\mathrm{m}}(1) & x_{\mathrm{m}}(2) & \cdots & x_{\mathrm{m}}(\mathrm{n})
\end{array}\right] \\
Y=\left[\begin{array}{c}
Y_{1} \\
Y_{2} \\
\vdots \\
Y_{\mathrm{m}}
\end{array}\right]=\left[\begin{array}{cccc}
y_{1}(1) & y_{1}(2) & \cdots & y_{1}(\mathrm{n}) \\
y_{2}(1) & y_{2}(2) & \cdots & y_{2}(\mathrm{n}) \\
\vdots & \vdots & \ddots & \vdots \\
y_{\mathrm{m}}(1) & y_{\mathrm{m}}(2) & \cdots & y_{\mathrm{m}}(\mathrm{n})
\end{array}\right]
\end{gathered}
$$

Step two: Matrix dimensionless. Due to the above factors have different dimensions and the numerical difference is large, which do not have comparability. So it is necessary to carry out changes in the value for $X_{i}$ and $Y_{i}$, through dimensionless processing to eliminate the influence of various factors dimension. Commonly used methods include initialization, equalization, and interval relative value and normalization methods. In this paper the method of interval relative value is used to convert the numerical to the interval $[0,1]$, transformation sequence can be obtained:

$$
\begin{aligned}
X_{l}^{\prime} & =\left[\begin{array}{llll}
X_{l}^{\prime}(1) & X_{l}^{\prime}(2) & \cdots & X_{l}^{\prime}(\mathrm{n})
\end{array}\right] \\
Y_{l}^{\prime} & =\left[\begin{array}{llll}
Y_{l}^{\prime}(1) & Y_{l}^{\prime}(2) & \cdots & Y_{l}^{\prime}(\mathrm{n})
\end{array}\right]
\end{aligned}
$$

Among them:

$$
X_{i}^{\prime}(j)=\frac{X_{i}(j)-\min _{j} X_{i}(j)}{\max _{j} X_{l}(j)-\min _{j} X_{l}(j)}
$$




$$
Y_{l}^{\prime}(j)=\frac{Y_{t}(j)-\min _{j} Y_{t}(j)}{\max _{j} Y_{t}(j)-\min _{j} Y_{l}(j)}
$$

Step three: Determine gray correlation difference information of the matrix. To obtain difference information and form a difference sequence matrix, then $X_{i}$ ' and $Y_{i}$ ' transformation is according to the following equation:

$$
\Delta_{i j}=\left|x_{i j}^{\prime}-y_{i j}^{\prime}\right|
$$

Find the maximum and minimum values in the difference sequence matrix $\Delta$ :

$$
\Delta_{\max }=\max \left(\Delta_{i j}\right) ; \Delta_{\min }=\min \left(\Delta_{i j}\right)
$$

Step Four: Find the correlation coefficient matrix. The relevance between comparative actors and reference factors is expressed by the correlation coefficient. The correlation coefficient can be calculated by the following formula:

$$
z_{l j}=\frac{\Delta_{\min }+\xi \Delta_{\max }}{\Delta_{i j}+\xi \Delta_{\max }}
$$

The $\xi$ is the resolution factor, taking a value between 0 and 1 , under normal circumstances take $\xi=0.5$.

Step five: Calculate correlation degree. Due to the large number of correlation coefficients, information is scattered and not easy to compare, correlation coefficient is therefore necessary to be gathered, finding out the average value as correlation degree to compare the relevance of influence factors. Correlation degree is calculated as follows:

$$
g_{i}=\frac{1}{n} \sum_{j=1}^{n} z_{i j}
$$

The size of the correlation degree is external performance between factors of interaction effect, which changes in the interval $[0,1]$. The greater correlation value indicates that this factor affect the stability of the landslide larger, which makes greater sensitivity; on the contrary, the less sensitive. To order the correlation degree is the sort of influence factors sensitivity, when it comes to correlation analysis, the different processing methods of sequence makes different calculated correlation degree value. But the sort of correlation degree is unchanged. It is worth noting that the size of the correlation degree does not mean that the contribution of the comparison sequences to reference sequences.

\section{Grey Correlation Model in the application of the Wen Zhutang landslide}

\subsection{Modeling and sensitivity analysis}

Wen Zhutang landslide belongs to a single planar landslide, this kind of rock slope is relatively common in the reservoir landslide, which often slides along the weak interlayer or weak structural surface. For this type of landslide, sensitivity analysis of the stability factor has a certain typicality and universal applicability. Since the average underground water level of landslide changes little, this paper selects four major influence factors foe the sensitivity analysis, including unit weight $(\gamma)$, cohesion (c), internal friction $(\varphi)$ and earthquake acceleration. Make landslide parameters of the basic values with $\mathrm{c}=18 \mathrm{kPa}, \varphi=23^{\circ}, \gamma=20 \mathrm{kN} / \mathrm{m}^{3}$, earthquake acceleration 0 , reservoir water level $98 \mathrm{~m}$. In the case of other parameters unchanged, change the value of one parameter. Use the rock landslide stability calculation method in < Specification of Design and Construction for Landslide Stabilization $>$ to find out the stability factor. Landslide stability coefficient $\mathrm{K}$ varies with the parameters are shown in Table 1 to Table 4.

Table 1. Relationship between the landslides stability factor $(\mathrm{K})$ and the unit weight $(\gamma)$

\begin{tabular}{ccccccc}
\hline$\gamma / \mathrm{kN} / \mathrm{m} 3$ & 16 & 18 & 20 & 22 & 24 & 26 \\
\hline $\mathrm{K}$ & 1.097 & 1.086 & 1.078 & 1.071 & 1.066 & 1.061 \\
\hline
\end{tabular}

Table 2. Relationship between the landslides stability factor (K) and the cohesion (c)

\begin{tabular}{ccccccc}
\hline $\mathrm{c} / \mathrm{kPa}$ & 12 & 14 & 16 & 18 & 20 & 22 \\
\hline $\mathrm{K}$ & 0.987 & 1.017 & 1.048 & 1.078 & 1.109 & 1.139 \\
\hline
\end{tabular}


Table 3. Relationship between the landslides stability factor $(K)$ and the angle of internal friction $(\varphi)$

\begin{tabular}{ccccccc}
\hline$\varphi /{ }^{\circ}$ & 17 & 19 & 21 & 23 & 25 & 27 \\
\hline $\mathrm{K}$ & 0.854 & 0.927 & 1.001 & 1.078 & 1.157 & 1.239 \\
\hline
\end{tabular}

Table 4. Relationship between the landslides stability factor $(\mathrm{K})$ and the earthquake acceleration

\begin{tabular}{ccccccc}
\hline $\mathrm{g}$ & 0 & 0.01 & 0.02 & 0.03 & 0.04 & 0.05 \\
\hline $\mathrm{K}$ & 1.078 & 1.04 & 1.021 & 0.988 & 0.964 & 0.944 \\
\hline
\end{tabular}

According to the data of the above tables, changes in the value of each factor are selected as a comparison matrix, and the corresponding landslide stability coefficient as a reference matrix. Establish the comparison matrix and reference matrix:

$$
\begin{aligned}
& X=\left[\begin{array}{l}
X_{1} \\
X_{2} \\
X_{3} \\
X_{4}
\end{array}\right]=\left[\begin{array}{cccccc}
16 & 18 & 20 & 22 & 24 & 26 \\
12 & 14 & 16 & 18 & 20 & 22 \\
17 & 19 & 21 & 23 & 25 & 27 \\
0 & 0.01 & 0.02 & 0.03 & 0.04 & 0.05
\end{array}\right] \\
& Y=\left[\begin{array}{l}
Y_{1} \\
Y_{2} \\
Y_{3} \\
Y_{4}
\end{array}\right]=\left[\begin{array}{llllll}
1.097 & 1.086 & 1.078 & 1.071 & 1.066 & 1.061 \\
0.987 & 1.017 & 1.048 & 1.078 & 1.109 & 1.139 \\
0.854 & 0.927 & 1.001 & 1.078 & 1.157 & 1.239 \\
1.078 & 1.040 & 1.021 & 0.988 & 0.964 & 0.944
\end{array}\right]
\end{aligned}
$$

Perform relative value of the interval through the above matrix and calculate the difference sequence matrix, which the correlation coefficient matrix can be obtained as follows:

$$
Z=\left[\begin{array}{cccccc}
0.3333 & 0.5028 & 0.8738 & 0.6081 & 0.4306 & 0.3333 \\
1 & 0.9948 & 0.9974 & 0.9974 & 0.9948 & 1 \\
1 & 0.9796 & 0.9649 & 0.9649 & 0.9747 & 1 \\
0.3333 & 0.4624 & 0.7443 & 0.6897 & 0.4494 & 0.3333
\end{array}\right]
$$

By the formula (10) can be obtained gray correlation degree is:

$\mathrm{G}=\left[\begin{array}{ll}0.5017 & 0.99740 .98070 .6021\end{array}\right]^{\mathrm{T}}$

Sorted by relevance degree shows the greatest influence of single planar landslide stability is c, $\varphi$ values and earthquake force, rock weight makes minimal impact. This also shows its emphasis on sliding body reduction plays the role of the landslide prevention is not significant.

\subsection{Treatment scheme}

Known from the analysis above, it is the mechanical parameters of mudstone interlayer play a major role for the stability of the landslide. In order to improve the landslide stability, reinforcement methods can be taken for improving the mechanical parameters of mudstone interlayer. Anchoring technology is a capable of improving the stress state of rock, improving the stability of rock reinforcement measure ${ }^{[13]}$. In particular restressed anchor technology has been widely used in various fields of geotechnical engineering reinforcement, including cracks, faults and high-steep slope with weak interlayer or weak structural plane. This method has been confirmed by a large number of engineering practices in improving the stress state, bearing capacity, stability and other aspects of rock and soil.

\section{Conclusions}

(1) Grey correlation analysis is well able to analyze the sensitivities of the various factors affecting the landslide. It is able to make the quantitative analysis results and clearly show the main factors and secondary factors, which provides reliable evaluation basis for prevention landslide engineering.

(2) The influence degree of multiple related influence factors of landslide stability is different. In this paper, the Wen Zhutang landslide are analyzed in the sensitive degree of various factors, the results show that the most sensitive parameter is shear strength. Therefore, during the landslide prevention work, as far as possible through combination of field test and laboratory test methods to 
obtain the true and accurate mechanical parameters of sliding surface. In the end, take the anchoring technology measures to improve the shear strength of the sliding surface for landslide prevention.

(3) This article involves the change of reservoir water level is small, the sensitivity of landslide stability during the gray correlation analysis was not considered. When in guiding other engineering instance, if the change of reservoir water level is larger, it will cause changes in shear strength, rock weight and other parameters. Characteristic changes of landslide stability in this case need to be considered under the multiple factors coupling.

(4) Long-term monitoring system for deformation and displacement of this landslide was not established, monitoring data is more able to provide reliable realistic basis to gray correlation analysis. Through the analysis of this paper, in terms of landslide natural geological environment and human engineering activities the prevention and control of landslides can be carried out in advance, thus playing a certain significance for the disaster prevention in reservoir area.

\section{Acknowledgments}

This work was supported by China Postdoctoral Science Foundation (Grant No. 2014M551453)

\section{References}

[1]Lin Lu-sheng, Feng Xia-ting, Bai Shi-wei, et al. Application of Artificial neural Network to Prediction of Sliding Slope [J]. Rock and Soil Mechanics, 2002, 23(4): 508-510.

[2] Zhang Wen, Chen Jian-ping, Wang Qing, An Yuke, Qian Xin, Xiang Liangjun, He Longxiang. Hazard assessment of regional large-scale debris flows based on combination weighting and extension methods [J]. Natural Hazards, 2013, 66, 1073-1100

[3]Zhang Zhuo-yuan, Wang Shi-tian, Wang Lan-sheng, et al. Principles of engineering geological analysis [M]. Beijing: Geological Publishing House, 2009.

[4] Zhang Wen, Li HuiZhong, Chen Jianping, Zhang Chen, Xu Liming, Sang Weifeng. Comprehensive hazard assessment and protection of debris flows along Jinsha River close to the Wudongde dam site in China [J]. Natural Hazards, 2011, 58: 459-477.

[5] Wang Yang, Yin Kun-long, An Guan-feng. Grey Correlation Analysis of Sensitive Factors of Landslide [J]. Rock and Soil Mechanics, 2004, 25(2): 91-93.

[6] Jing Pei-Deng, Fan Fu-song, Song Yi-liang, et al. Grey Correlation Analysis of Slope Sensitivity Based on Uniform Design [J]. Safety and Environmental Engineering, 2011, 18(2): 116-118.

[7] Wang Jun-Qing, Li Jing ,Li Qi, et al. Analysis of Influence Factors of High Slope Stability of Loess : Taking the Baojixia Water Division Project for Example [J]. Rock and Soil Mechanics, 2009, 30(7): 2114-2118.

[8] Liu Jin-Ying, Yang Tian-Xing, Li Ming, et al. A Weight Absolute Grey Correlation Degree and It's Application in Evaluation of Water Quality Miyun Reservoir [J]. Journal of Jilin University (Earth Science Edition), 2005, 35(1): 54-58.

[9]Chen Zhi-Bo, Jian Wen-Bin. Sensibility Analysis of Slopes Stability Based on Grey Correlation Analysis [J]. Journal of Disaster Prevention and Mitigation Engineering, 2006, 26(4): 473-477.

[10]Chen Xin-ming, Luo Guo-yu. Grey System Analysis and Evaluation of Slope Stability Based on Experience [ J]. Chinese Journal of Geotechnical Engineering, 1999, 21(5): 638-641.

[11] Wang Xue-meng, zhang Ji-zhong, Wang Rong. The Gray System Analysis and Practical Calculation Procedures [M]. Wuhan: Huazhong University of Science and Technology Press, 2001.

[12] Deng Ju-long. The Foundation of Grey Theory [M] .Wuhan: Huazhong Science and Technology Press, 2002.

[13] Zhu Jie-wang, Zhuang Hua-ze , et al. Research on Reinforcement Technique of Slope with Large Dimensions[J]. Journal of Engineering Geology, 2008, 16 ( 03 ): 365-370

[14]Nie Lei, Xu Yan, Dai Shulin , et al. Slope Engineering[M]. BeiJing: Science Press, 2010, 9. 